\title{
Quantum Chemical Modeling of Molecule 6-Aminopenicillanic Acid (6-Apa), of Model of 6-Apa-Phosgene and 6-Apa-Acetyl Chloride
}

\author{
A. K. Boshkayeva, R. A. Omarova, A. S. Kozhamzharova, A. D. Masakbayev, L. Kiyekbayeva, \\ G. Ibadullayeva, E. M. Bisenbaev, Z. Olatayeva, Zh. Muhametkan, M. Kudaybergenova, \\ R. Abdykalykov
}

Pharmaceutical Faculty, Asfendiyarov Kazakh National Medical University, Almaty, Republic of Kazakhstan

Email address:

kenes65@mail.ru (A. K. Boshkayeva),omarova-r@list.ru (R. A. Omarova), assel_kozhamzharova@mail.ru (A. S. Kozhamzharova), abai.masakbayev@mail.ru (A. D. Masakbayev), lashynk@mail.ru (L. Kiyekbayeva), arujan-d@mail.ru (G. Ibadullayeva), bissenbayev1941@mail.ru (E. M. Bisenbaev), zukhra.olataeva@mail.ru (Z. Olatayeva), juparm@mail.ru (Zh. Muhametkan) poppi.32@mail.ru (M. Kudaybergenova)raha_92kz@mail.ru (R. Abdykalykov)

\section{To cite this article:}

A. K. Boshkayeva, R. A. Omarova, A. S. Kozhamzharova, A. D. Masakbayev, L. Kiyekbayeva, G. Ibadullayeva, E. M. Bisenbaev, Z. Olatayeva, Zh. Muhametkan, M. Kudaybergenova, R. Abdykalykov. Quantum Chemical Modeling of Molecule 6-Aminopenicillanic Acid (6-Apa), of Model of 6-Apa-Phosgene and 6-Apa-Acetyl Chloride. Clinical Medicine Research. Special Issue: Development of Drugs: Computer Simulation, Chemical Synthesis and Clinical Trials. Vol. 5, No. 2-2, 2016, pp. 8-10. doi: 10.11648/j.cmr.s.2016050202.12

Received: February 17, 2016; Accepted: March 17, 2016; Published: August 2, 2016

\begin{abstract}
For the first time a quantum-chemical method PM3 (Parametric Method 3) in semi empirical approach appreciated geometric and electronic parameters of the new acylated form the molecules 6-APA (6-aminopenicillanic acid), identified patterns of change according to the nature of the acylating agent. In general, the process of acylation of the molecule 6-APA by acylating agents (phosgene and acetyl chloride) leads to much larger changes in new systems of electronic structure than in its spatial structure. In this electronic structure analysis indicates that the acylation of 6-APA of the molecule both acylating agents leads to formation of more stable in the plan hydrolytic cleavage of form of this acid. This fact is a prediction of the characters and is positive for future synthesis of new antibiotics on the basis of 6-APA. Significant absolute value and the sign of the negative enthalpy of formation calculated by PM3, for both systems 6-APA-phosgene 6-APA-acetyl chloride indicate their high thermodynamic stability. The most stable in terms of the thermodynamic system is the 6-APA-acetyl chloride.
\end{abstract}

Keywords: Semiempirical Quantum Chemical Method, Schrödinger Equation, Parametric Method 3, Modeling, 6-Aminopenicillanic Acid

\section{Introduction}

Antibiotics of penicillin group are the potential inhibitors biosynthesis of cell wall, and the selectivity of their action on the bacterium cell is usually defined by structural features as bacterial cell and peculiarities of the structure of antibiotics $[1,2]$.

An important aspect of the study of the biological activity of new semisynthetic penicillins is to establish its relationship with the peculiarities of electronic and spatial (geometric) structure of molecules of 6-APA (6aminopenicillanic acid), having new substituents [3]. The most correct geometric parameters of molecular structure now it can only be obtained by using X-ray analysis method. However, application of this method to most molecular objects connected with experimental dif-ficulties of producing test samples in the form of single crystals and with their aggression and hygroscopic. Therefore, to obtain the most appropriate to the real characteristics of spatial and electronic structure [4] is now widely used quantum chemical calculations of model molecules.

To establish the features of spatial and electronic structure, receiving the energy parameters of the initial reagents and microbiological synthesis products have been carried out 
quantum chemical calculations of these objects by semi empirical quantum chemical method PM3 (Parametric Method 3). In the first stage of this research have been identified optimal method of calculation in connection with which have been tried out three semi empirical quantum chemical methods: MNDO, AM1 (Austin Model 1), PM3 (Parametric Method 3) included in the software package Hyper Chem of version 7, and the method $\mathrm{PM}^{*}$, part of a software package MOPAC of version 7. Semi empirical method is a method of valence approximation, ie take into account the valence electrons and atomic orbitals (AO) of the valence shells. The ultimate aim of this calculation method consists in solving the time-independent Schrödinger equation $[5,6]$, which describes the state of the microsystem with two physical quantities: the total energy $\mathrm{E}$ of the system and the wave function $\Psi$, which is a function of a coordinate or pulses: $\hat{\mathrm{H}} \Psi=\mathrm{E} \Psi$. As a result, as calculated quantum chemical method was selected the PM3 method.

\section{The Experimental Part}

The spatial structure of the initial model molecules of 6-APA, two acylating agents (molecules of phosgene and molecules of acetyl chloride), and the two acylated forms of 6-APA was optimized PM3 method and were calculated parameters of the electronic structure and their energy characteristics $[7,8,10]$. As a result have been obtained the geometric models, examples of which are shown in Figures 1-3.
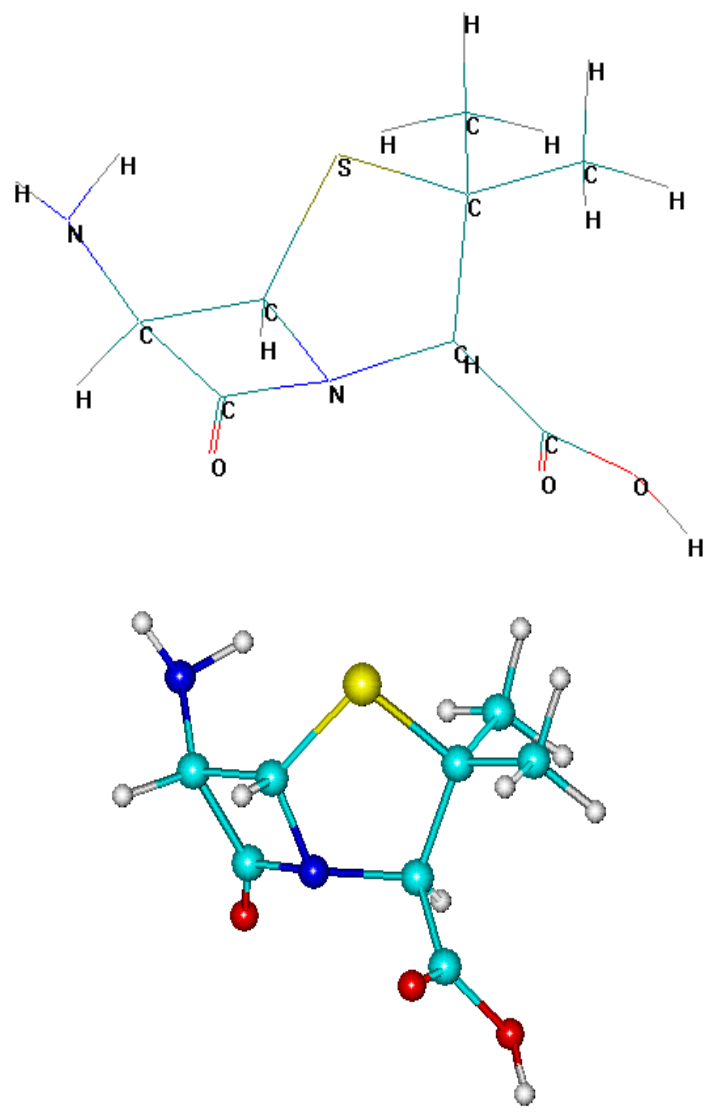

Figure 1. Geometric model of a molecule of 6-APA.
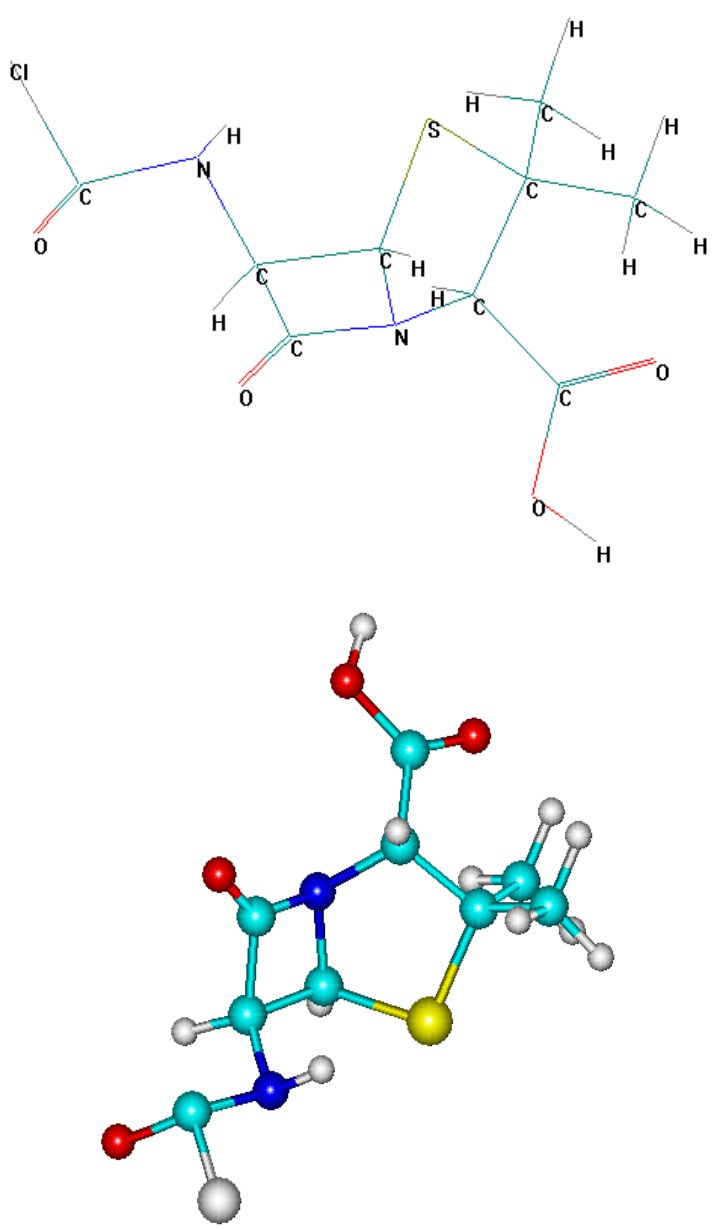

Figure 2. Geometric model of a molecule of 6-APA-phosgene.
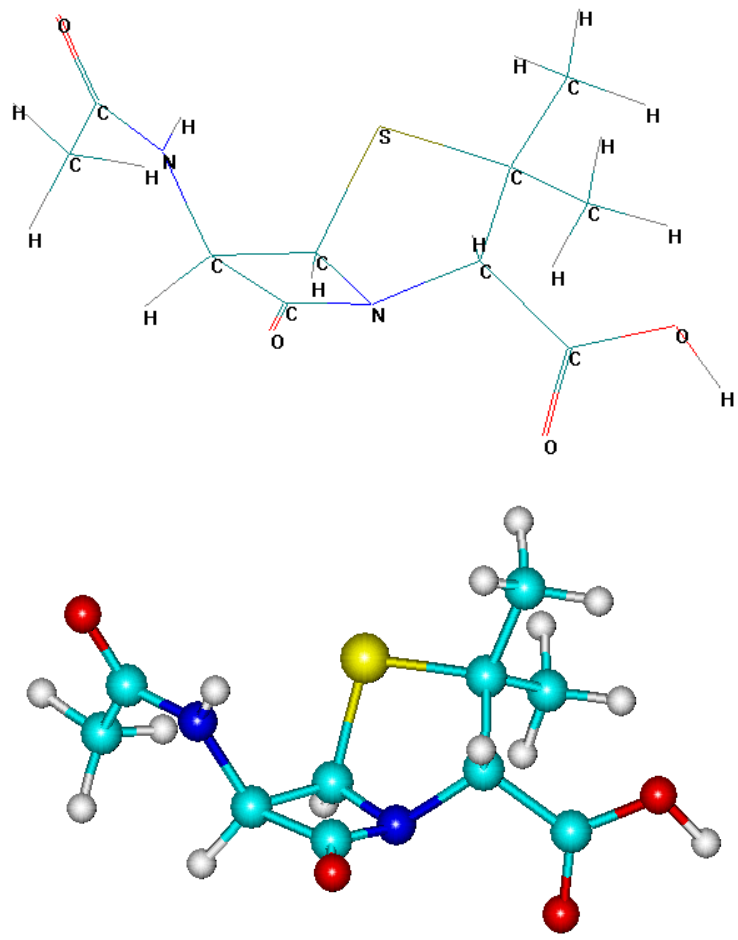

Figure 3. Geometric model of a molecule of 6-APA-acetyl chloride. 
Thus been found that the guy of electron density is observed for b-lactam ring, and greater electron-donating ability of the oxygen atom bonded to this ring in the molecule 6-APA. This suggests its role in the formation of a prevailing HOMO (highest occupied molecular orbital) responsible for the reactivity of b-lactam ring and the associated oxygen atom.

\section{Discussion of Results}

During the acylation of 6-APA molecules of phosgene and acetyl chloride comparative analysis of change of geometrical parameters allowed to make following general conclusion about the influence of the spatial structure of the acylating agent: in the space a more compact molecule of phosgene leads to a noticeable change in the bond lengths in the molecule of 6-APA. Comparative analysis of the electronic parameters of the model of the molecule 6-APA and its systems with molecules of acetyl chloride and phosgene has shown that in general the acylation process results in a much larger change in the electronic structure of the new system compared to the parent molecule of 6-APA.

On the basis of values of difference between the energies of the frontier orbitals (highest occupied and the lowest unoccupied molecular orbital) was established character of the molecule acylation of 6-APA process, which as shown by the results of studies is the orbital controlled reaction.

Has been found that a significant absolute value and in sign to the negative enthalpy of formation calculated by the PM3 method, show high thermodynamic stability of the acylated forms of 6-APA. The most stable in terms of the thermodynamic system is the 6-APA-acetyl chloride.

Chemical interaction between the molecules of 6-APA and acetyl chloride leads to a noticeable change of the geometric parameters. Moreover to a greater extent it relates to the molecule fragments that are not directly related to the interaction center.

So the interatomic distance between the nitrogen atom of the amino group and its associated carbon atom in the heterocycle model molecule 6-APA still 1,47 $\AA$. In the product of its reaction with acetyl chloride is almost constant value. While the interatomic distances between the nitrogen and carbon atoms in the heterocyclic ring system are reduced considerably. In the heterocyclic system interatomic distances are changed between carbon atoms: they either increase or decrease depending on location of the $\mathrm{C}-\mathrm{C}$ bond to the nitrogen hetero atoms. $\mathrm{C}-\mathrm{O}$ distance equal to a molecule of acetyl chloride 1,20 $\AA$, and its interaction with the 6-APA is lengthened twice the distance between the chlorine and carbon atoms is reduced to $1,60 \AA$.

\section{Conclusions}

Thus, the quantum chemical study shows that interaction between molecules of 6-APA and acetyl chloride leads to a change in the electronic and geometric characteristics and indicates the unsaturation of reaction formed product, which appear new electron donor centers.

The results of quantum chemical studies have allowed on the stage of theoretical quantum chemical calculations to choose the most promising molecules of acylated form of 6APA, which is a system of 6-APA-acetyl chloride. More precisely this form were prepared using the method of microbiological synthesis $[11,12]$.

\section{References}

[1] VG Belikov Pharmaceutical Chemistry. In 2 parts: Textbook / V. G. Belikov - 4th edition, revised and enlarged. - M.: MEDpress-Inform, 2007. - $624 \mathrm{p}$.

[2] Egorov NS Basics of theory of antibiotics. - M.: Medicine, 2004. - 528 p.

[3] Omarova RA, Boshkayeva AK Prospects for the development of research in the development of original drugs // Collection of materials of the International Scientific-Practical Conference. "Pharmacy: Current status, achievements and prospects". - Almaty, 2010 - P. 58-61.

[4] Gribov LA, Mushtakova SP Quantum Chemistry. - M.: Gardariki. - 1999.

[5] Zhidomirov GM, Bagaturyants AA, Abronin IA Applied quantum chemistry. - M.: Chemistry, 1979. - 340 p.

[6] T. Clark Computer chemistry. - M.: Mir, 1990. - 383 p.

[7] Omarova RA, Boshkayeva AK Geometric and electronic characteristics of 6-aminopenicillanic acid and its acetyl derivatives // Abstracts of the 1st All-Russia Scientific Conference "Methods of studying the composition and structure of functional materials", on 11-16 October 2009, Novosibirsk. - P.310.

[8] Omarova RA, Boshkayeva AK, Abdullin KA, Nokerbek Sh Geometric and electronic parameters of the molecule 6aminopenicillanic acid according to quantum chemical calculations // Abstracts of the XII International scientific conference "Science and education - the leading factor of strategy" Kazakhstan-2030”, Karaganda, 2009.

[9] Omarova RA, Boshkayeva AK Features enzymatic ways of obtaining beta-lactam antibiotics // Collection of materials of the International Scientific-Practical Conference. "Pharmacy: Current status, achievements and prospects". - Almaty, 2010 - P. 25-32.

[10] Omarova RA, Boshkayeva AK Quantum-chemical modeling of the acylation of 6-aminopenicillanic acid // Proceedings of the international scientific-practical conference "Pharmacy: Current status, achievements and prospects". - Almaty, 2010 P. 144-150.

[11] Boshkayeva AK, Omarova RA, Shakeev SS, Masakbayev AD Isolation of the enzyme penicillin acylase and determination of its activity // Proceedings of the international scientificpractical conference. "Pharmacy: current state, achievements and prospects", devoted to the 80th anniversary of Asfendiyarov Kazakh National Medical University: Almaty, 2011. - P.62-66.

[12] Omarova RA, Boshkayeva AK, Shakeev SS Biotechnological aspects of producing enzymes // "Family Health Century XXİ century". Proceedings of the XV International Scientific Conference, April 30 - 07 May 2011, Torremolinos, Spain. P. $85-87$ 